\title{
Long non-coding RNAs and hepatocellular carcinoma (Review)
}

\author{
FU-JUN YU ${ }^{1}$, JIAN-JIAN ZHENG ${ }^{2}$, PEI-HONG DONG ${ }^{3}$ and XIAO-MING FAN ${ }^{1}$ \\ ${ }^{1}$ Department of Gastroenterology, Jinshan Hospital of Fudan University, Jinshan, Shanghai 201508; \\ ${ }^{2}$ Wenzhou Key Laboratory of Surgery, ${ }^{3}$ Department of Infectious Diseases, The First Affiliated Hospital \\ of Wenzhou Medical University, Wenzhou, Zhejiang 325000, P.R. China
}

Received March 5, 2014; Accepted September 13, 2014

DOI: $10.3892 / \mathrm{mco} .2014 .429$

\begin{abstract}
Recent advances in next-generation sequencing technology in transcriptome analysis have helped identify numerous non-coding RNAs. The long non-coding RNA (lncRNA) is commonly defined as an RNA molecule with a length of $200 \mathrm{bp}-100 \mathrm{kbp}$ that lacks protein-coding potential. LncRNAs play a critical role in the regulation of gene expression, including chromatin modification, transcription and post-transcriptional processing. It has been confirmed that dysregulation of IncRNAs is associated with a number of human diseases, particularly tumors. In this study, we focused on the most extensively investigated lncRNAs in hepatocellular carcinoma (HCC). The biological functions and molecular mechanisms of the majority of IncRNAs have yet to be investigated. The improved knowledge on lncRNAs in HCC may help identify lncRNAs that may be used as novel prognostic markers and therapeutic targets.
\end{abstract}

\section{Contents}

1. Introduction

2. Characteristics of IncRNAs

3. Dysregulation of IncRNAs in HCC

4. Conclusions

\section{Introduction}

With the development of deep sequencing and DNA tiling array technology, an increasing number of investigators are focusing

Correspondence to: Professor Xiao-Ming Fan, Department of Gastroenterology, Jinshan Hospital of Fudan University, 1508 Longhang Road, Jinshan, Shanghai 201508, P.R. China E-mail: ktsqdph@163.com

Key words: long non-coding RNA, hepatocellular carcinoma, metastasis associated lung adenocarcinoma transcript 1, HOX antisense intergenic RNA, H19, maternally expressed gene 3, microvascular invasion in HCC, highly upregulated in liver cancer, downregulated expression by $\mathrm{HBx}$, high expression in $\mathrm{HCC}$ on non-coding RNAs (ncRNAs) that are not translated into protein and the number of articles on ncRNAs is increasing exponentially (1-3). It was estimated that $\sim 70 \%$ of the human genome is pervasively transcribed; however, protein-coding genes account for $<2 \%$ of the human genome (4). Consequently, abundant ncRNAs are transcribed from the human genome, including small interfering RNAs (siRNAs), microRNAs (miRNAs), PIWI-interacting RNAs and long ncRNAs (lncRNAs) (5-7). Extensive studies have been conducted on siRNAs and miRNAs and their molecular functions have been elucidated (8-10). However, our understanding of the involvement of lncRNAs in diseases remains limited. Recent studies revealed that lncRNAs play a pivotal role in the regulation of gene expression, such as chromatin modification, transcription and post-transcriptional processing (11-13).

In this study, we aimed to focus on some critical, well-known lncRNAs in hepatocellular carcinoma (HCC) and briefly outline their known functions and possible underlying molecular mechanisms, as well as their potential application as therapeutic targets or biomarkers.

\section{Characteristics of IncRNAs}

The most common definition of lncRNA is an RNA molecule with a length of $200 \mathrm{bp}-100 \mathrm{kbp}$ that lacks protein-coding potential (14). However, this simplistic definition may be associated with several problems (15). For example, the cut-off of 200 nucleotides was factitiously selected and was not based on functional meaning. In addition, lncRNAs may contain an open reading frame longer than 100 amino acids. However, polypeptides shorter than 100 amino acids may also be functional in organisms and are not by-products of canonical proteins. These problems suggest our limited understanding of lncRNAs and how difficult it is to determine a definition.

The majority of lncRNAs are transcribed by RNA polymerase II and then undergo co-transcriptional modifications, including polyadenylation and pre-RNA splicing (16). LncRNAs participate in several biological processes, such as epigenetic regulation, transcriptional and post-transcriptional regulation, processing of small RNAs and other regulatory functions (17-20).

Establishing a widely accepted classification of lncRNAs is a challenging task. There are currently five broad categories of lncRNAs, namely i) sense or ii) antisense, when overlapping one or more exons of another transcript on the same or opposite 
strand, respectively; iii) bidirectional, when the expression of lncRNA and a neighboring coding transcript on the opposite strand is initiated in close genomic proximity; iv) intronic, when the lncRNA is derived from an intron of a splicing transcript; and v) intergenic, when it lies in the genomic interval between two genes $(21,22)$.

To facilitate the search for IncRNAs, the currently available online databases including ncRNAs are listed in Table I. These databases were retrieved from GenBank annotations or from published manuscripts. Some of these databases have been experimentally verified, others are merely computational predictions or have been annotated as ncRNAs according to the predicted length of their open reading frames.

\section{Dysregulation of IncRNAs in HCC}

The number of studies on lncRNAs is soaring unexpectedly (23-25). In this study, we aimed to summarize the potential functions and molecular mechanisms of the most widely investigated lncRNAs in HCC.

Metastasis-associated lung adenocarcinoma transcript 1 (MALAT1). MALAT1, also referred to as nuclear-enriched abundant transcript 2, is a nuclear lincRNA $>8,000$ nt that is transcribed from chromosome 11q13 (26). The highly preserved level of MALAT1 in numerous species indicates its functional significance (25). MALAT1 has been shown to modulate primary transcripts transcriptionally or post-transcriptionally $(27,28)$.

Lai et al (29) reported that MALAT1 was increased in vitro and in vivo. In addition, analysis of multivariate data demonstrated that MALAT1 level was an independent prognostic factor for HCC recurrence. More importantly, the higher expression of MALAT1 was also associated with shortened patient disease-free survival following liver transplantation. These results suggest that treatment by targeting MALAT1 may be of clinical value by selectively eliminating diffuse or residual cancer cells following surgery. Silencing of MALAT1 by siRNA in HepG2 cells may significantly decrease cell viability, inhibit motility and invasiveness and sensitize cells to multi-stimuli-induced apoptosis.

HOX antisense intergenic RNA (HOTAIR). Another lncRNA implicated in cancer metastasis is referred to as HOTAIR, is encoded in antisense direction from the HOXC gene cluster and acts in trans to regulate HOXD genes through interaction and recruitment of the polycomb repressive complex 2 (PRC2) to induce transcriptional silencing (30). Notably, pull-down assays with $\mathrm{PRC} 2$ revealed a direct and specific interaction with HOTAIR, which was an unexpected novel finding.

High levels of HOTAIR are mostly correlated with poor patient survival rate and tumor recurrence (31-33). Ishibashi et al (34) reported that high expression of HOTAIR was identified in primary HCCs in 13 of 64 patients. Patients with high HOTAIR expression exhibited significantly poorer prognoses and a larger primary tumor size compared to those with low HOTAIR expression. Furthermore, application of human HOTAIR to liver cancer cells demonstrated that HOTAIR induced a more rapid proliferation when compared to control samples.
Table I. Public ncRNA databases.

Website

Name

http://biobases.ibch.poznan.pl/ncRNA/

http://www.noncode.org

Noncode

http://research.imb.uq.edu.au/rnadb

RNAdb

http://www.ncrna.org

fRNA

http://jsm-research.imb.uq.edu.au/nred/

cgi-bin/ncrnadb.pl

NRED

http://www.lncrnadb.org

LncRNAdb

http://rfam.sanger.ac.uk

Rfam

ncRNA, non-coding RNA.

Geng et al (35) demonstrated that knockdown of HOTAIR in the Bel7402 HCC cell line led to the reduction of matrix metalloproteinase-9 and vascular endothelial growth factor proteins, which are critical for cell motility and metastasis. Their study also revealed that there was a obvious association between HOTAIR level and lymph node metastasis. Thus, HOTAIR lincRNA may be a potential biomarker for the presence of lymph node metastasis in HCC. Yang et al (36) reported that the HOTAIR level in HCC was higher compared to that in adjacent non-cancerous tissues. Additionally, a high level of HOTAIR was an independent prognostic marker for predicting HCC recurrence in liver transplantation patients. Furthermore, patients with a higher level of HOTAIR exhibited significantly shorter recurrence-free survival. Similar to breast cancer, the inhibition of HOTAIR by siRNA in a liver cancer cell line decreased cell viability and invasion, sensitized cancer cells to tumor necrosis factor $\alpha$-induced apoptosis and improved the sensitivity of cancer cells to cisplatin and doxorubicin.

H19. Oncofetal H19 had been extensively investigated in cancer biology, even before research was focused on lncRNA $(37,38)$. H19 is a paternally imprinted gene which locates at chromosome 11p15.5. H19 is highly expressed during embryonic development, but is repressed right after birth in most tissues $(39,40)$. H19 is highly expressed during tumorigenesis and was shown to possess tumorigenic properties in all types of tissues $(37,41-43)$.

Matouk et al (44) demonstrated that H19 was excessively produced in a human $\mathrm{HCC}$, even more than the traditional HCC marker $\alpha$-fetoprotein. Therefore, an approach based on H19 knokdown may be an efficient method of targeting tumor cells. The results of that study suggested that HCC tumors transfected with H19 siRNA exhibited significant inhibition of tumor growth and, in certain cases, there was complete inhibition of tumor formation. There was a $82 \%$ decrease of mean tumor weights and mean tumor volumes in the two transfected cell lines. By contrast, Zhang et al (45) demonstrated that $\mathrm{H} 19$ was downregulated in intratumoral HCC tissues (T) when compared to peritumoral tissues (L). In addition, the Kaplan-Meier analysis revealed that HCC patients with low $\mathrm{T} / \mathrm{L}$ ratios exhibited a worse prognosis compared to those with high ratios. H19 inhibited HCC metastasis and epithelial-to-mesenchymal transition. More 
importantly, H19 stimulated the miR-200 family by inducing histone acetylation, thus inhibiting the rate of tumor metastasis in advanced-stage HCC. The results of that study provided novel insights to the mechanism underlying the modulation of the level of small ncRNAs by lncRNAs. Yoshimizu et al (46) demonstrated that $\mathrm{H} 19$ regulated the timing of appearance of SV40-induced hepatocellular carcinoma using in vivo murine models of tumorigenesis. Therefore, it was concluded that H19 exerts a tumor suppressor effect in mice.

In summary, the above contradictory results indicate that the precise functions of $\mathrm{H} 19$ remain to be further investigated.

Maternally expressed gene 3 (MEG3). Another extensively investigated lncRNA is MEG3, which locates on chromosome 14q32. MEG3 belongs to the DLK1-MEG3 imprinting locus, containing at least three paternal protein-coding genes and abundant maternal ncRNAs $(47,48)$. The gene expression in this locus is firmly regulated by at least two differentially methylated regions (DMRs), namely the intergenic DMR and the MEG3-DMR (49)

MEG3 plays a crucial role in cell development and growth $(50,51)$. In humans, MEG3 is highly expressed in normal tissues and downregulation of MEG3 has been identified in a number of human tumors $(50,52)$. Additionally, upregulation of MEG3 inhibits in vitro tumor cell proliferation. Consequently, it was concluded that MEG3 is a tumor suppressor gene (53).

Braconi et al (54) reported that MEG3 was decreased by 210-fold in HCC tumor tissues compared to non-malignant hepatocytes. Enforced expression of MEG3 in HCC cells notably inhibited cell growth and increased apoptosis. Furthermore, hypermethylation of MEG3 promoter was detected by methylation-specific PCR and the level of MEG3 was increased with siRNA treatment of DNA methyltransferase (DNMT) 1 and 3b. More importantly, miR-29, which is able to regulate DNMT 1 and 3, increased the level of MEG3. These findings highlighted the interrelationship between two classes of non-coding RNAs, namely miRNAs and lncRNAs, and the epigenetic modulation of gene expression.

In addition, Anwar et al (55) suggested that the DLK1-MEG3 locus was continually deregulated in HCC. Knockdown of DNMT1 in HCC cells resulted in a reduction of MEG3-DMR methylation and a subsequent increase in the MEG3 level.

Microvascular invasion in HCC (MVIH). MVIH is located in the intron of the RPS24 gene and encodes a protein that belongs to the S24E family of ribosomal proteins (56). Despite the location of MVIH within the RPS24 gene, these two genes are transcribed separately, indicating that MVIH may play an independent role in biological behavior (57).

Yuan et al (58) reported an lncRNA termed MVIH that was increased in tumor tissues compared to the corresponding non-cancerous tissues and was associated with microvascular invasion of HCC. Furthermore, MVIH was considered to be an independent risk factor predictive of poor recurrence-free survival. The authors of that study also observed that MVIH was able to boost tumor growth and intrahepatic metastasis in vivo. The suppression of phosphoglycerate kinase 1 secretion by MVIH resulted in activation of angiogenesis in vitro and in vivo.
Highly upregulated in liver cancer (HULC). HULC is a 500-nt lncRNA located on chromosome 6p24.3 (59). Compared to non-neoplastic liver tissues, high expression of HULC was observed in HCC tissues (60). Xie et al (61) found that HULC IncRNA was detected with higher frequency in the plasma of HCC patients when compared to healthy controls. Higher HULC detection rates were also observed in the plasma of patients with higher Edmondson grades or with hepatitis B virus (HBV)-positive status. These findings suggested for the first time that the level of HULC in the plasma may be used as a novel, non-invasive biomarker for the diagnosis and/or prognosis of HCC. Interestingly, in other tumors, no obvious difference was observed in the expression of HULC between the tumor and normal tissues (62). In addition, high level of HULC was detected in metastatic liver nodules from colon cancer, but not in the primary colorectal carcinoma specimen and corresponding normal tissues. High level of HULC was also detected in liver metastases but not in lymph nodes, demonstrating its specificity to any malignant cells located in the liver (62).

Wang et al (63) provided evidence that HULC was a self-amplifying, auto-regulatory loop through inhibition of miRNA-372. There was a binding site of cAMP response element-binding protein (CREB) in the proximal promoter region of HULC (from -67 to $-53 \mathrm{nt}$ ). Activation of the protein kinase A pathway was implicated in the upregulation of HULC. HULC may function as an endogenous 'sponge', downregulating the expression and activity of miR-372. Suppression of miR-372 resulted in reducing the translational inhibition of its target gene, cAMP-dependent protein kinase catalytic subunit beta, which in turn increased the phosphorylation of CREB. This regulatory loop demonstrates the latent interactions between lncRNAs and miRNAs and increases the complexity of the gene regulation network.

Liu et al (64) indicated that the variant genotypes of rs7763881 were significantly correlated with reduced HCC risk in a dominant genetic model. However, there was no significant association between single-nucleotide polymorphisms and HBV clearance. Therefore, the authors concluded that the variant genotypes of rs7763881 in HULC may contribute to the decreased susceptibility to HCC in HBV chronic carriers.

$\mathrm{Du}$ et al (65) revealed that HULC participated in HBx-mediated HCC. The expression of HULC was positively associated with the level of HBx in clinical HCC samples. Moreover, the luciferase reporter gene assay and chromatin immunoprecipitation assay indicated that $\mathrm{HBx}$ improved the activation of the HULC promoter via CREB. Finally, it was confirmed that the upregulation of HULC by HBx increased the proliferation of HCC cells through inhibiting p18. Taken together, these findings provide novel insight into the roles of lncRNAs in HBx-related HCC.

Other IncRNAs in HCC. Recent studies revealed that several other lncRNAs are dysregulated in HCC. Huang et al (66) reported that downregulated expression by $\mathrm{HBx}$ (termed lncRNA-Dreh) was able to decrease HCC growth and function as a tumor suppressor in the progression of HBV-related HCC. It was further observed that IncRNA-Dreh altered the normal cytoskeleton structure to suppress tumor metastasis by targeting the intermediate filament protein vimentin. 
In addition, lncRNA-Dreh was significantly associated with poor prognosis of HCC. In summary, these results may provide a strategy for the development of lncRNA-based targeted methods for the treatment of HBV-related HCC. Yang et al (67) revealed that the level of lncRNA high expression in HCC (termed lncRNA-HEIH) in HBV-related HCC was markedly associated with recurrence and was an independent prognostic biomarker for survival. The authors of that study also manifested that lncRNA-HEIH played a pivotal role in G0/G1 arrest. It was further demonstrated that lncRNA-HEIH was correlated with enhancer of zeste homolog 2 (EZH2) and that this correlation was required for the inhibition of EZH2 target genes. Taken together, these results reveal that IncRNA-HEIH is an oncogenic lncRNA that promotes tumor development and, thus, it may act as a critical regulatory factor in HCC progression. Yang et al (68) indicated that uc $002 \mathrm{mbe} .2$ exhibited the most notable changes among these differentially expressed lncRNAs in HCC cell lines upon trichostatin A (TSA) treatment. Moreover, the TSA-induced uc002mbe. 2 expression was positively associated with the apoptotic effect of TSA on HCC cells. Furthermore, a reduction in the level of uc002mbe. 2 significantly decreased TSA-induced apoptosis of Huh7 cells, consequently unmasking that the TSA-induced apoptosis of HCC cells is uc002mbe.2-dependent and the low expression of uc002mbe. 2 may be associated with liver carcinogenesis.

\section{Conclusions}

High-throughput sequencing has helped demonstrate the dysregulation of numerous lncRNAs in HCC. However, our current knowledge of lncRNAs compared to miRNAs is limited. Further investigation is required to elucidate the biological functions and molecular characteristics of IncRNAs in HCC.

\section{Acknowledgements}

This study was supported by grants from the Science and Technology Commission of Shanghai Municipality (no. 11ZR1405700), the Key Clinical Disciplines Construction of Shanghai Municipality (no. ZK2012B20), the National Natural Science Foundation of China (nos. 81000176/H0317 and 81100292/H0317), the Zhejiang Provincial Natural Science Foundation of China (no. Y2110634), the Wang Bao-En Liver Fibrosis Foundation (no. 20120127) and the Wenzhou Municipal Science and Technology Bureau (no. Y20110033).

\section{References}

1. Zheng J, Lin Z, Dong P, et al: Activation of hepatic stellate cells is suppressed by microRNA-150. Int J Mol Med 32: 17-24, 2013.

2. Zheng J, Dong P, Gao S, Wang N and Yu F: High expression of serum miR-17-5p associated with poor prognosis in patients with hepatocellular carcinoma. Hepatogastroenterology 60: 549-552, 2013.

3. Zheng J, Wu C, Lin Z, et al: Curcumin up-regulates phosphatase and tensin homologue deleted on chromosome 10 through microRNA-mediated control of DNA methylation - a novel mechanism suppressing liver fibrosis. FEBS J 281: 88-103, 2014.
4. Huang B and Zhang R: Regulatory non-coding RNAs: revolutionizing the RNA world. Mol Biol Rep Feb 19, 2014 (Epub ahead of print).

5. Gehrau RC, Mas VR, Villamil FG, et al: MicroRNA signature at the time of clinical HCV recurrence associates with aggressive fibrosis progression post-liver transplantation. Am J Transplant 13: 729-737, 2013.

6. Dong P, Yu F, Fan X, Lin Z, Chen Y and Li J: Inhibition of ATIR by shRNA prevents collagen synthesis in hepatic stellate cells. Mol Cell Biochem 344: 195-202, 2010.

7. Gomes AQ, Nolasco S, Soares H. Non-coding RNAs: multi-tasking molecules in the cell. Int J Mol Sci. 14(8):16010-16039. 2013.

8. Gao SM, Xing CY, Chen CQ, Lin SS, Dong PH and Yu FJ: miR-15a and miR-16-1 inhibit the proliferation of leukemic cells by down-regulating WT1 protein level. J Exp Clin Cancer Res 30: 110, 2011.

9. Yu FJ, Dong PH,Fan XF, LinZ, Chen YP and Li J: Downregulation of angiotensin II by shRNA reduces collagen synthesis in hepatic stellate cells. Int J Mol Med 25: 801-806, 2010.

10. Chen SL, Zheng MH, Yang T, Song M and Chen YP: Disparate profiles of dys-regulated miRNAs in activated hepatic stellate cells. Hepatology 57: 1285-1286, 2013.

11. Kitagawa M, Kotake Y and Ohhata T: Long noncoding RNAs involved in cancer development and cell fate determination. Curr Drug Targets 13: 1616-1621, 2012.

12. Yoon JH, Abdelmohsen K, Srikantan S, et al: LincRNA-p21 suppresses target mRNA translation. Mol Cell 47: 648-655, 2012.

13. Yoon JH, Abdelmohsen K and Gorospe M: Posttranscriptional gene regulation by long noncoding RNA. J Mol Biol 425: 3723-3730, 2013.

14. Zhang Q, Chen CY, Yedavalli VS and Jeang KT: NEAT1 long noncoding RNA and paraspeckle bodies modulate HIV-1 post-transcriptional expression. MBio 4: e00596-e00512, 2013.

15. Qiu MT, Hu JW, Yin R and Xu L: Long noncoding RNA: an emerging paradigm of cancer research. Tumour Biol 34: 613-620, 2013.

16. Danko CG, Hah N, Luo X, et al: Signaling pathways differentially affect RNA polymerase II initiation, pausing, and elongation rate in cells. Mol Cell 50: 212-222, 2013.

17. Moran VA, Perera RJ and Khalil AM: Emerging functional and mechanistic paradigms of mammalian long non-coding RNAs. Nucleic Acids Res 40: 6391-6400, 2012.

18. Gibb EA, Vucic EA, Enfield KS, et al: Human cancer long non-coding RNA transcriptomes. PLoS One 6: e25915, 2011.

19. Bussemakers MJ, van Bokhoven A, Verhaegh GW, et al: DD3: a new prostate-specific gene, highly overexpressed in prostate cancer. Cancer Res 59: 5975-5979, 1999.

20. van Devondervoort II, Gordebeke PM, Khoshab N, et al: Long non-coding RNAs in neurodevelopmental disorders. Front Mol Neurosci 6: 53, 2013.

21. Gutschner T and Diederichs S: The hallmarks of cancer: a long non-coding RNA point of view. RNA Biol 9: 703-719, 2012.

22. Weikard R, Hadlich F and Kuehn C: Identification of novel transcripts and noncoding RNAs in bovine skin by deep next generation sequencing. BMC Genomics 14: 789, 2013.

23. Zhang Y, Yang L and Chen LL: Life without A tail: new formats of long noncoding RNAs. Int J Biochem Cell Biol Oct 26, 2013 (Epub ahead of print) doi: 10.1016/j.biocel.2013.10.009.

24. Nadal-Ribelles M, Solé C, Xu Z, Steinmetz LM, de Nadal E and Posas F: Control of Cdc28 CDK1 by a stress-induced lncRNA. Mol Cell 53: 549-561, 2014.

25. Imamura K, Imamachi N, Akizuki G, et al: Long noncoding RNA NEAT1-dependent SFPQ relocation from promoter region to paraspeckle mediates IL8 expression upon immune stimuli. Mol Cell 53: 393-406, 2014.

26. $\mathrm{Li} \mathrm{CH}$ and Chen $\mathrm{Y}$ : Targeting long non-coding RNAs in cancers: progress and prospects. Int J Biochem Cell Biol 45: 1895-1910, 2013.

27. Yang F, Yi F, Han X, Du Q and Liang Z: MALAT-1 interacts with hnRNP C in cell cycle regulation. J FEBS Lett 587: 3175-3181, 2013.

28. Shi X, Sun M, Liu H, Yao Y and Song Y: Long non-coding RNAs: a new frontier in the study of human diseases. Cancer Lett 339: 159-166, 2013.

29. Lai MC, Yang Z, Zhou L, et al: Long non-coding RNA MALAT-1 overexpression predicts tumor recurrence of hepatocellular carcinoma after liver transplantation. Med Oncol 29: 1810-1816, 2012.

30. Rinn JL, Kertesz M, Wang JK, et al: Functional demarcation of active and silent chromatin domains in human HOX loci by noncoding RNAs. Cell 129: 1311-1323, 2007. 
31. Sørensen KP, Thomassen M, Tan Q, et al: Long non-coding RNA HOTAIR is an independent prognostic marker of metastasis in estrogen receptor-positive primary breast cancer. Breast Cancer Res Treat 142: 529-536, 2013.

32. Zhang JX, Han L, Bao ZS, et al: HOTAIR, a cell cycle-associated long noncoding RNA and a strong predictor of survival, is preferentially expressed in classical and mesenchymal glioma. Neuro Oncol 15: 1595-1603, 2013.

33. Chen FJ, Sun M, Li SQ, et al: Upregulation of the long non-coding RNA HOTAIR promotes esophageal squamous cell carcinoma metastasis and poor prognosis. Mol Carcinog 52: 908-915, 2013.

34. Ishibashi M, Kogo R, Shibata K, et al: Clinical significance of the expression of long non-coding RNA HOTAIR in primary hepatocellular carcinoma. Oncol Rep 29: 946-950, 2013.

35. Geng YJ, Xie SL, Li Q, Ma J and Wang GY: Large intervening non-coding RNA HOTAIR is associated with hepatocellular carcinoma progression. J Int Med Res 39: 2119-2128, 2011.

36. Yang Z, Zhou L, Wu LM, et al: Overexpression of long non-coding RNA HOTAIR predicts tumor recurrence in hepatocellular carcinoma patients following liver transplantation. Ann Surg Oncol 18: 1243-1250, 2011.

37. Matouk I, Raveh E, Ohana P, et al: The increasing complexity of the oncofetal h19 gene locus: functional dissection and therapeutic intervention. Int J Mol Sci 14: 4298-4316, 2013.

38. Ratajczak MZ: Igf2-H19, an imprinted tandem gene, is an important regulator of embryonic development, a guardian of proliferation of adult pluripotent stem cells, a regulator of longevity, and a 'passkey' to cancerogenesis. Folia Histochem Cytobiol 50: 171-179, 2012

39. Necsulea A, Soumillon M, Warnefors M, et al: The evolution of lncRNA repertoires and expression patterns in tetrapods. Nature 505: 635-640, 2014

40. Monnier P, Martinet C, Pontis J, Stancheva I, Ait-Si-Ali S and Dandolo L: H19 lncRNA controls gene expression of the imprinted gene network by recruiting MBD1. Proc Natl Acad Sci USA 110: 20693-20698, 2013.

41. Yu LL, Chang K, Lu LS, et al: Lentivirus-mediated RNA interference targeting the H19 gene inhibits cell proliferation and apoptosis in human choriocarcinoma cell line JAR. BMC Cell Biol 14: 26, 2013.

42. Gao T, He B, Pan Y, et al: H19 DMR methylation correlates to the progression of esophageal squamous cell carcinoma through IGF2 imprinting pathway. Clin Transl Oncol 16: 410-417, 2013.

43. Luo M, Li Z, Wang W, Zeng Y, Liu Z and Qiu J: Upregulated H19 contributes to bladder cancer cell proliferation by regulating ID2 expression. FEBS J 280: 1709-1716, 2013.

44. Matouk IJ, DeGroot N, Mezan S, et al: The H19 non-coding RNA is essential for human tumor growth. PLoS One 2: e845, 2007.

45. Zhang L, Yang F, Yuan JH, et al: Epigenetic activation of the MiR-200 family contributes to H19-mediated metastasis suppression in hepatocellular carcinoma. Carcinogenesis 34: 577-586, 2013

46. Yoshimizu T, Miroglio A, Ripoche MA, et al: The H19 locus acts in vivo as a tumor suppressor. Proc Natl Acad Sci USA 105: $12417-12422,2008$.

47. Kameswaran V, Bramswig NC, McKenna LB, et al: Epigenetic regulation of the DLK1-MEG3 microRNA cluster in human type 2 diabetic islets. Cell Metab 19: 135-145, 2014.

48. Tran DA, Bai AY, Singh P, Wu X and Szabo PE: Characterization of the imprinting signature of mouse embryo fibroblasts by RNA deep sequencing. Nucleic Acids Res 42: 1772-1783, 2014

49. Benetatos L, Voulgaris E and Vartholomatos G: DLK1-MEG3 imprinted domain microRNAs in cancer biology. Crit Rev Eukaryot Gene Expr 22: 1-15, 2012.

50. Qin R, Chen Z, Ding Y, Hao J, Hu J and Guo F: Long non-coding RNA MEG3 inhibits the proliferation of cervical carcinoma cells through the induction of cell cycle arrest and apoptosis Neoplasma 60: 486-492, 2013.

51. Ying L, Huang Y, Chen H, et al: Downregulated MEG3 activates autophagy and increases cell proliferation in bladder cancer. Mol Biosyst 9: 407-411, 2013.
52. Balik V, Srovnal J, Sulla I, et al: MEG3: a novel long noncoding potentially tumour-suppressing RNA in meningiomas. J Neurooncol 112: 1-8, 2013.

53. Zhou Y, Zhang X and Klibanski A: MEG3 noncoding RNA: a tumor suppressor. J Mol Endocrinol 48: R45-R53, 2012.

54. Braconi C, Kogure T, Valeri N, et al: microRNA-29 can regulate expression of the long non-coding RNA gene MEG3 in hepatocellular cancer. Oncogene 30: 4750-4756, 2011.

55. Anwar SL, Krech T, Hasemeier B, et al: Loss of imprinting and allelic switching at the DLK1-MEG3 locus in human hepatocellular carcinoma. PLoS One 7: e49462, 2012.

56. He Y, Meng XM, Huang C, et al: Long noncoding RNAs: novel insights into hepatocelluar carcinoma. Cancer Lett 344: 20-27, 2014.

57. Avellino AM, Grant GA, Harris AB, Wallace SK and Shaw CM Recurrent intracranial Masson's vegetant intravascular hemangioendothelioma. Case report and review of the literature. J Neurosurg 91: 308-312, 1999.

58. Yuan SX, Yang F, Yang Y, et al: Long noncoding RNA associated with microvascular invasion in hepatocellular carcinoma promotes angiogenesis and serves as a predictor for hepatocellular carcinoma patients' poor recurrence-free survival after hepatectomy. Hepatology 56: 2231-2241, 2012.

59. Zhao Y, Guo Q, Chen J, Hu J, Wang S and Sun Y: Role of long non-coding RNA HULC in cell proliferation, apoptosis and tumor metastasis of gastric cancer: a clinical and in vitro investigation. Oncol Rep 31: 358-364, 2014.

60. Panzitt K, Tschernatsch MM, Guelly C, et al: Characterization of HULC, a novel gene with striking up-regulation in hepatocellular carcinoma, as noncoding RNA. Gastroenterology 132: 330-342, 2007.

61. Xie H, Ma H and Zhou D: Plasma HULC as a promising novel biomarker for the detection of hepatocellular carcinoma. Biomed Res Int 2013: 136106, 2013

62. Matouk IJ, Abbasi I, Hochberg A, Galun E, Dweik H and Akkawi M: Highly upregulated in liver cancer noncoding RNA is overexpressed in hepatic colorectal metastasis. Eur J Gastroenterol Hepatol 21: 688-692, 2009.

63. Wang J, Liu X, Wu H, et al: CREB up-regulates long non-coding RNA, HULC expression through interaction with microRNA-372 in liver cancer. Nucleic Acids Res 38: 5366-5383, 2010.

64. Liu Y, Pan S, Liu L, et al: A genetic variant in long non-coding RNA HULC contributes to risk of HBV-related hepatocellular carcinoma in a Chinese population. PLoS One 7: e35145, 2012.

65. Du Y, Kong G, You X, et al: Elevation of highly up-regulated in liver cancer (HULC) by hepatitis B virus X protein promotes hepatoma cell proliferation via down-regulating p18. J Biol Chem 287: 26302-26311, 2012.

66. Huang JF, Guo YJ, Zhao CX, et al: Hepatitis B virus X protein (HBx)-related long noncoding RNA (lncRNA) down-regulated expression by HBx (Dreh) inhibits hepatocellular carcinoma metastasis by targeting the intermediate filament protein vimentin. Hepatology 57: 1882-1892, 2013.

67. Yang F, Zhang L, Huo XS, et al: Long noncoding RNA high expression in hepatocellular carcinoma facilitates tumor growth through enhancer of zeste homolog 2 in humans. Hepatology 54: $1679-1689,2011$

68. Yang H, Zhong Y, Xie H, et al: Induction of the liver cancerdown-regulated long noncoding RNA uc002mbe. 2 mediates trichostatin-induced apoptosis of liver cancer cells. Biochem Pharmacol 85: 1761-1769, 2013. 